\title{
Development of Facial Expression Training System
}

\author{
Kyoko Ito ${ }^{1}$, Hiroyuki Kurose ${ }^{2}$, Ai Takami ${ }^{2}$,and Shogo Nishida ${ }^{2}$ \\ ${ }^{1}$ Senri Expo Park 1-1, Suita, 5650826 Osaka, Japan \\ 2 1-3, Machikaneyama-cho, Toyonaka, 5608531 Osaka, Japan \\ \{ito, kurose, takami, nishida\}@nishilab.sys.es.osaka-u.ac.jp
}

\begin{abstract}
The human's facial expression plays an important role as media that visually transmit feelings and the intention. To express the facial expression freely, the action of muscles of facial expression forms the expression is very important. "Facial expression training" is recently paid to attention, and is a training of the face to make a good facial expression. There is a training method by moving the expression muscle of the face. One of the purposes to train the facial expression is to achieve your own ideal facial expression. In this study, it aims at a support of the effective process for facial expression training to achieve the target expression using computer, as a self training system. And, an interface for users to select a target facial expression and a whole development of an effective expression training system is proposed, as a first step toward an effective facial expression training system.
\end{abstract}

Keywords: Facial expression, Facial expression training system, Muscles of facial expression, Nonverbal communication.

\section{Introduction}

In the human communication, nonverbal information such as the facial expression, the gesture, the voice plays an important role [1]. Especially, the facial expression is very important media that visually transmit feelings and the intention [2][3]. A study shows that more than 50 percent of the impression of communications is transmitted based on the visual information [4].

As for the expression, the person who expresses a facial expression cannot see the expression directly. It is important to understand your real expression and to image your target facial expression in order to express your own ideal facial expression. The muscles of facial expression play an important role for expressing the human facial expression.

Facial expression training is recently paid to attention to make your facial expression better [5][6][7][8]. Some exercises of the part of the face and the expression muscle are performed for facial expression training. The muscles of facial expression are strengthened by training, and the facial expression is softened, and your own ideal facial expression is expressed. Facial expression training can be utilized effectively by not only the communications scene in daily life but also the scene of the business skill and rehabilitation. Facial expression training has some 
forms. One is seminar style with a trainer. One of the others is self training using training books or the information on the Internet as a guide. Some seminars are very expensive, and time and space are restricted. On the other hand, when self training is performed, it is difficult to image your target facial expression clearly alone, and to compare the expression the ideal facial expression with your present one.

In this study, it aims at the proposal of an effective expression training system using the computer toward the achievement of your target facial expression. As the first step, an interface to select the target facial expression is proposed. And, the expression training system including the target expression selection interface is developed.

There is a facial expression training system with a virtual mirror as a previous study [9], from the viewpoint of a support system of facial expression training using a computer. Virtual mirror is a facial expression training system that displays your facial expression emphasizing the feature with a virtual mirror. This study is different from virtual mirror from the viewpoint of selecting the target facial expression of actual face.

\section{Facial Expression Training System to Achieve Target Facial Expression}

\subsection{Support Process}

In this study, the following processes are considered as a step of facial expression training to achieve the target facial expression.

(1) The target facial expression is imaged.

(2) The current facial expression is expressed, aiming at expressing the target facial expression.

(3) The current facial expression is compared with a target facial expression.

(4) The muscles of facial expression for improvement are trained.

In this study, above processes are supported with a computer. And, it aims at the development of the facial expression training system to achieve the target facial expression. The supporting method with a computer is considered for each process, as follows:

(1) Support to make target facial expression

(2) Support to recognize current expression

(3) Support to compare current facial expression with target facial expression

(4) Support to understand muscles of facial expression for which training is necessary

A main point of this study is (1) support to make target facial expression, and a user interface is proposed. A proposal of the user interface is described as follows.

\subsection{Support to Make Target Facial Expression}

In this study, it pays attention to the following viewpoints to make the target facial expression. 
(a) Your own face is used.

(b) Your target facial expression can be made to correspond to the movement of real human's face, and made freely.

As for (a), it is important to make the target facial expression by using your real face, because human face is different from each one, and your facial expression corresponds to your features of face.

As to (b), it is necessary to make the target expression that can be actually expressed based on anatomic movement using your face. And, it is important to make freely satisfied target facial expression.

From the viewpoints, a user interface to select the target facial expression is considered. And, the following approaches of two stages are considered so that the user may select a target facial expression as satisfactory.

- First stage: Rough expression selection

- Second stage: Detailed expression selection

At the first stage, for the user not to worry about a detailed part, and to select the target expression intuitively, the interface shown in Figure 1 is proposed.

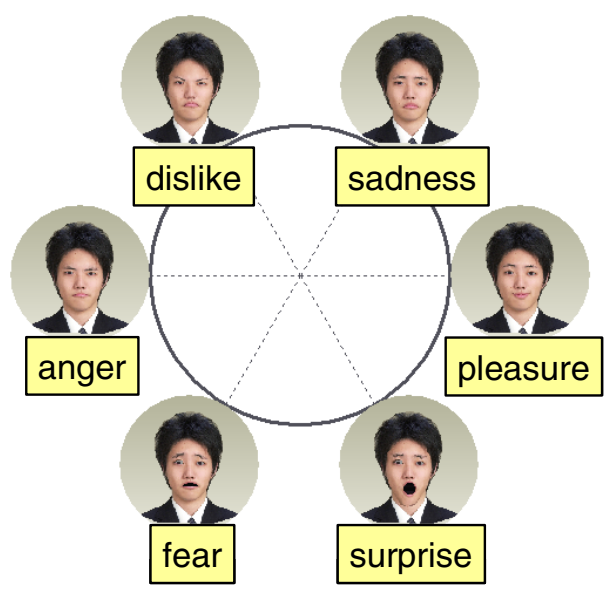

Fig. 1. Interface for selection of target facial expression

As for figure 1, six face images of facial expression made by your face are arranged outside of a big circle at the center [10]. These six images are corresponded to six basic facial expressions of please, surprise, dislike, anger, fear, and sadness. And, the mixture expression of two images and the expression corresponding to strength can be selected by selecting one point on the circle at the center.

In the second stage, a user interface for a user to control a detailed part so that the user is satisfied is considered. In this stage, action unit (AU) $\mathrm{n}$ is used from the previous researches on facial expression parts by Ekman et al [11]. The elements of each part corresponding to AU can be set in detail. The parts are eyebrows, eyes, cheeks, mouths, and mandibles. AUs of 3, 5, 2, 10 and 5 are used in the each part. And, 25 kinds of AU are used in total. 


\section{Design and Development of Facial Expression Training System}

A facial expression training system including above mentioned processes and the interface for selection of target facial expression is developed.

First of all, the personal computer camera is used to take a current facial expression of the user.

Figure 2 shows the hardware setup. Visual $\mathrm{C}++6.0$ is used as a software for the development. To fit your face with a wire frame model, FaceFit [12] is used. The facial expression training system has been developed [13][14], and it was named "iFace". The utilization of iFace has the following procedures.

(1) User registration

(2) Selection of target facial expression

(3) Expression of current facial expression

(4) Comparison of current facial expression and target facial expression

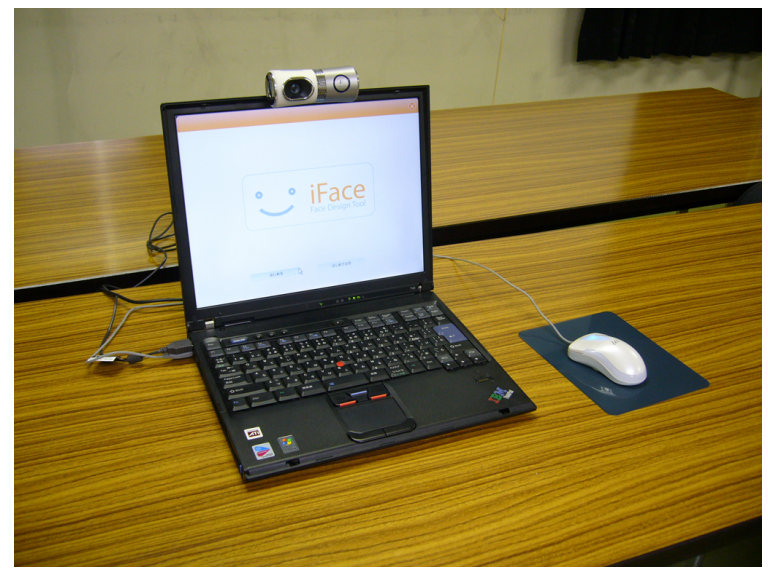

Fig. 2. Hardware setup

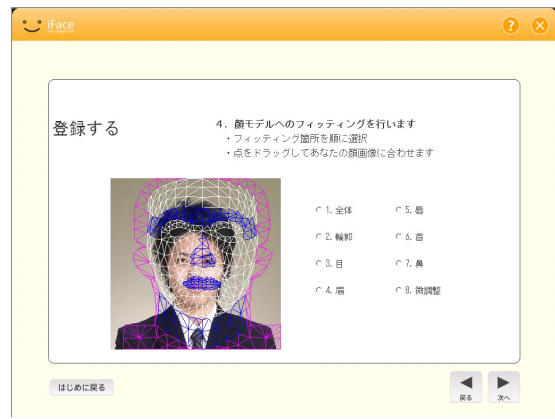

Fig. 3. A screen example of user registration for fitting 


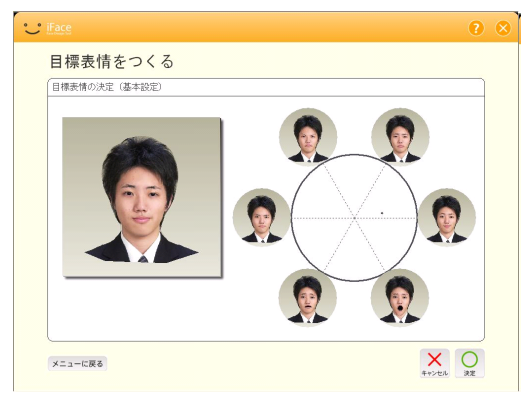

Fig. 4. For rough setting

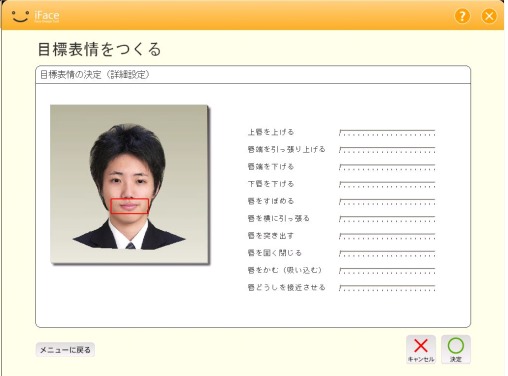

Fig. 5. For detailed setting

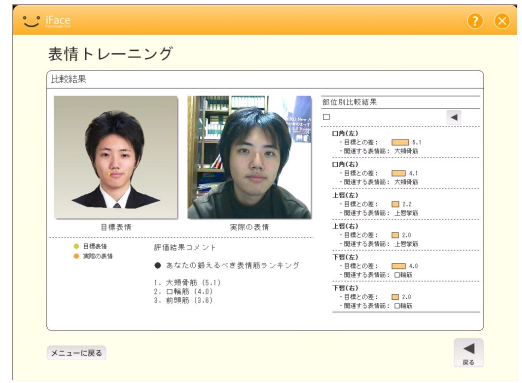

Fig. 6. A screen example of comparison between current and target

Figure 3 shows a screen of the user registration for the fitting. The screens for a rough setting and a detailed setting are shown in figure 4 and figure 5 respectively, for the target facial expression selection. Figure 6 shows the comparison result presentation screen between a current facial expression and target facial expression.

\section{Evaluation Experiment of Facial Expression Training System}

\subsection{Purposes}

To examine the effectiveness of the target facial expression selection interface and the facial expression training system, the evaluation experiment is conducted. Concretely, it aims to examine the following points through the evaluation experiment.

- Effectiveness in target facial expression selection interface for facial expression training system

- Possibility of facial expression training system 


\subsection{Methods}

A. Procedure of experiment

(1) The target facial expression is selected by using the facial expression training system by a user.

(2) A user aims at the realization of the target facial expression that the user selected, and a current facial expression of the user is expressed.

(3) The current facial expression is compared with the target facial expression, and the results are presented.

One user makes two target facial expressions. One is the ideal smile of the user, and another the free choice of the user. A current facial expression is expressed three times by the one target. So, six expression of the current facial expression are tried in total.

B. Experiment participant

- 12 female (dentist)

C. Methods of analysis

The results of the questionnaire before and after the experiment are used. In addition, the log data during using the facial expression training system are used.

\subsection{Results}

- Effectiveness in target facial expression selection interface

Table 1. Results of questionnaire for effectiveness in target facial expression selection interface

\begin{tabular}{c|l|c}
\hline$\#$ & \multicolumn{1}{c}{ Item of questionnaire } & Avg. \\
\hline 1 & $\begin{array}{l}\text { Did you satisfactorily come to the target expression? } \\
\text { (The first) }\end{array}$ & -0.3 \\
\hline 2 & $\begin{array}{l}\text { Did you satisfactorily come to the target expression? } \\
\text { (The second) }\end{array}$ & 1.7 \\
\hline 3 & $\begin{array}{l}\text { Was the facial expression selection interface intuitive and } \\
\text { comprehensible? }\end{array}$ & 2.2 \\
\hline 4 & Did you feel the synthesized facial expression natural? & -0.3 \\
\hline
\end{tabular}

Table 1 shows the results of the questionnaire for effectiveness in target facial expression selection interface. The questionnaire is seven point scale of +3 to -3 . As to the average score of the satisfaction rating, the first time (\#1) and the second time (\#2) are -0.3 and 1.7. It is shown that the satisfaction rating to the selection of the smile is lower than that of the free choice.

In addition, the time required to select the target facial expression is shown in Figure 7 separately for a rough setting and a detailed setting at the smile selection. In the comparison with a rough setting and a detailed setting, it is shown that there were a lot of people who spend time in a detailed setting. 


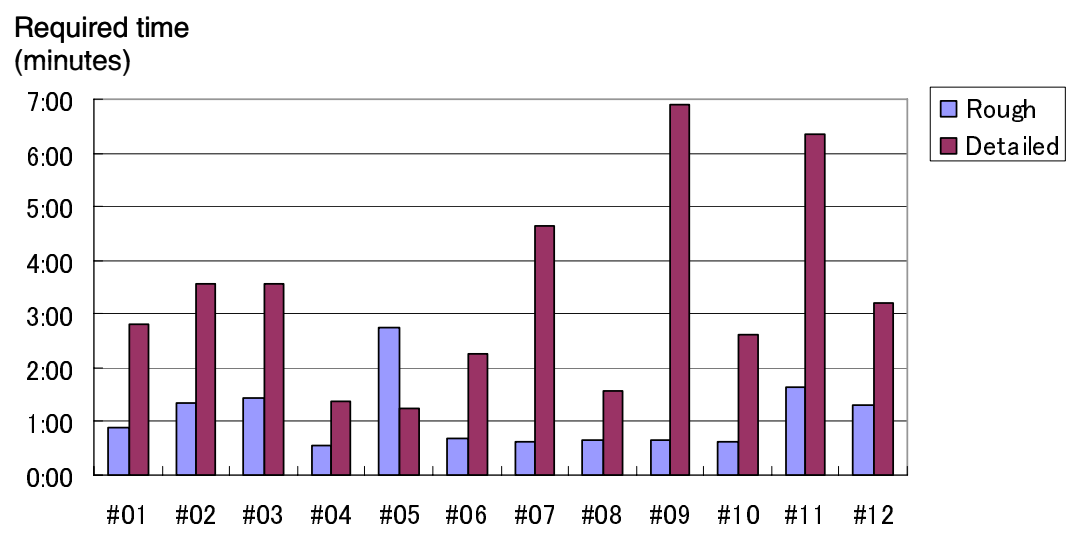

Participant \#

Fig. 7. Required time for rough and detailed selection of target facial expression by each user

- Possibility of facial expression training system

Table 2. Results of questionnaire for possiblity of facial expression training system

\begin{tabular}{c|c|c}
\hline$\#$ & Item of questionnaire & Avg. \\
\hline 5 & $\begin{array}{c}\text { Was the training method of deciding the target facial expression } \\
\text { concrete and comprehensible? }\end{array}$ & 2.6 \\
\hline 6 & $\begin{array}{c}\text { Was it easy to express your actual expression by comparing the } \\
\text { current facial expression with the target facial expression? }\end{array}$ & 2.5 \\
\hline 7 & Do you daily want to use the facial expression training system? & 1.8 \\
\hline
\end{tabular}

Table 2 shows results of the questionnaire for possibility of facial expression training system. The questionnaire is seven point scale of +3 to -3 . It is shown that the facial expression training system by this study was positively evaluated.

The following comments were obtained from a free description.

- I think that they can use it to undergo rehabilitation of the face paralytic.

- My motivation for training of the facial expression goes up by making the target facial expression.

\section{Conclusion}

In this study, a target facial expression selection interface for facial expression training system, and a facial expression training system were proposed and developed. Twelve female dentists used the facial expression training system, and the evaluation and the opinion about the facial expression training system was obtained. 
In the future, the improvement of the target facial expression selection interface and the comparison of a current and target facial expression will be tried, and it will aim at the development of an effective facial expression training system that can be actually used for various purposes.

\section{References}

[1] Kurokawa, T.: Nonverbal interface. Ohmsha, Ltd, Tokyo (1994) (In Japanese)

[2] Yoshikawa, S.: Facial expression as a media in body and computer, Kyoritsu Shuppan Co., Ltd, pp. 376-388 (2001) (In Japanese)

[3] Uchida, T.: Function of facial expression, Bungeisha, Co., Ltd (2006) (In Japanese)

[4] Mehrabian, A.: Silent messages, Implicit Communication of Emotions and Attitudes, 2nd edn. Wadsworth Pub. Co (1981)

[5] Inudou, F.: Facening Official Site (February 2007) (In Japanese) http://www.facening.com/

[6] Inudou, F.: Facening. Seishun Publishing Co., Ltd, Tokyo (1997) (In Japanese)

[7] COBS ONLINE Business good face by facial muscles training (February 2007) (In Japanese) http://cobs.jp/skillup/face/index.html

[8] Practice of Facial Expression (February 2007) (In Japanese) http://www.nikkeibp.co.jp/style/biz/associe/expression/

[9] Miwa, S., Katayori, H., Inokuchi, M. (eds.): Virtual mirror: Proposal of Facial expression training system by face image data processing. Proc. 43th conference of the institute of system, control and information engineers, pp. 343-344 (1999)

[10] Schlosberg, H.: The description of facial expression in terms of two dimensions. Journal of Experimental Psychology 44 (1952)

[11] Ekman, P., Friesen, W.V.: The Facial Action Coding System. Consulting Psychologists Press (1978)

[12] Galatea Project: (February 2007) (In Japanese) http://hil.t.u-tokyo.ac.jp/ galatea/indexjp.html

[13] Parke, F.I.: Techniques for facial animation, New Trends in Animationand Visualization, pp. 229-241 (1991)

[14] Waters, K.: A Muscle Model for Animating Three dimensional FacialExpression, Computer Graphics. SIGGRAPH' 87 2(4), 17-24 (1987) 